\title{
Numerical study of battle damaged two-dimensional wings
}

\author{
S. Djellal, T. Azzam, M. Djellab \& K. Lakkaichi \\ Fluid Mechanics Laboratory Polytechnical School Bordj El Bahri, \\ Alger, Algeria
}

\begin{abstract}
The flow around a NACA $64_{1}-412$ airfoil with circle damage is numerically investigated using the Ansys Fluent package. Several diameter values are considered for the damage which is located at either quarter or mid chord. The numerical domain is covered with a multiblock structured hexagonal grid consisting of 344,680 cells in the undamaged case and 351,540 cells in the damaged case. Inside the damage hole, a structured tetragonal mesh is used. Turbulence effects are taken into account via the $\mathrm{k}-\varepsilon$ model. The results show that the presence of the damage hole decreases the lift coefficient and increases the drag coefficient, resulting in a loss of airfoil performance (fineness decrease). The numerical simulations show that the flow through the damage corresponds either to a weak or a strong jet. In the first case an attached wake forms giving the smallest change in the force coefficients whereas the second case shows a separated wake with a reverse flow giving the highest force coefficient change. The present paper also compares the structure of the damage through flow with previously published experimental results. Finally, the numerical solution is qualitatively and quantitatively validated using available experimental results.

Keywords: damage, drag, lift, strong jet, weak jet, undamaged.
\end{abstract}

\section{Introduction}

The survivability of an aircraft is becoming one of the key aircraft design requirements [1] and is dependant upon its vulnerability to damage caused by a variety of threats. Published works to date in this field focus mainly on twodimensional wings. 
The first study by Irwin at Loughborough University was extensively reported $[2,3]$ and by many other authors; Render et al. [4], Mani and Render [5] and Djellal and Ouibrahim [6]. Recently Saaedi et al. [7] published a numerical study in this field.

In this investigation, we are interested in the aerodynamic performance losses caused to a two dimensional wing by the impact of battle damage. For this matter, we investigate by means of numerical methods the undamaged case and damaged case by considering the variation of the hole diameter and its chordwise position.

In this work, we analyse the flow field inside the damage hole and also around the undamaged and damaged aerofoil. In order to validate the numerical results, obtained values have been compared with those of experimental investigations led by the previous authors $[2,5,6,8]$.

\section{Damage modelling}

The most common type of damage used in simulations is the circular hole [6]. The study of other shapes reported by Mani and Render [5] has not shown noticeable differences.

Damage size can be expressed in terms of a percentage diameter $d$ to the local chord length $c$. The range of damage sizes varies from 0.1 to $0.4 \mathrm{c}$ [2] and, for our simulations, two diameters, $0.2,0.3$, have been considered and both were located at mid span.

NACA $64_{1}-412$ aerofoil experimented firstly by Render and his colleagues serves as the model's cross section. The aerofoil's chord and the wing's span are $200 \mathrm{~mm}$ and $450 \mathrm{~mm}$ respectively (Fig. 1).

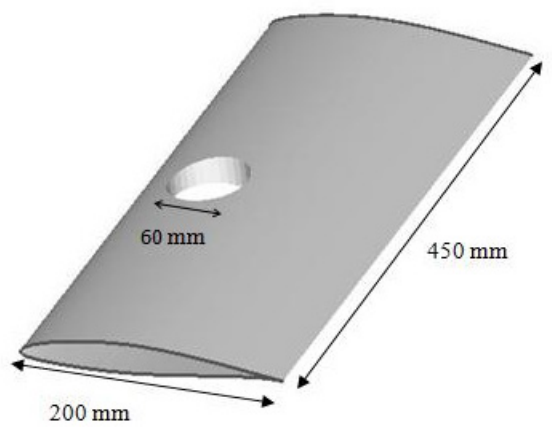

Figure 1: $\quad$ Circle damaged wing at quarter chord.

We only considered wing damaged at the quarter and the mid-chord, because they produced the greatest adverse influence on aerodynamic performance [2]. 


\section{Modelling and grid generation}

The numerical domain is made discrete by two approaches: (1) structured tetragonal cells for circle damage (2) multiblock structured hexagonal cells are applied for the remainder damaged aerofoil. The finite volume discretisation method is used in 'Fluent' numerical code.

Following a suitable study of grid sensitivity, Number of cells applied in this numerical domain is 344,680 cells in the undamaged case and 351,540 cells in the damaged case. It is worth mentioning that the used structured mesh that is able to capture the boundary layer was a complicated and time consuming process. The multiblock approach generated in Gambit allows via the interface condition to reduce the cells number and consequently the time process.

The results did not show a major difference between aerodynamic coefficients, pressure distribution or other phenomena in three grid types. The calculations with such amount of grids are made possible with the use of an ordinary 2-core processors, $2.5 \mathrm{GHz}$ computer.

\section{Boundary conditions}

Five types of boundaries are used in this model: Velocity inlet, pressure outlet, symmetry, interface and wall. Dimensions of numerical domain are considered big enough to damp turbulences before reaching the domain boundaries. The boundary conditions for lower and upper surface of the aerofoil are considered as solid wall.

The wind tunnel used for the experiments is a low subsonic low turbulence $(0 \cdot 1 \%$ of turbulence intensity) wind tunnel at Loughborough University and the dimensions of the test section are: $45 \mathrm{~cm} \times 45 \mathrm{~cm} \times 1 \cdot 2 \mathrm{~m}$. The test section velocity is $35 \mathrm{~ms}^{-1}$.

\section{Numerical solution characteristics}

The flow in this analysis is assumed to be steady, incompressible, and turbulent. For modelling the viscous turbulent flow, $k-\varepsilon$ turbulence model is applied. SIMPLE algorithm is applied to solve these two equations. Standard wall functions are also used for the areas close to wall (parietal distance $\mathrm{y}^{+}=40$ ). For the turbulence parameters at the inlet and the outlet, we use the method which gives the intensity $(0.1 \%)$ [2] and the viscosity ratio $v_{t} / v=5$ after tests. This value is chosen for a stall detection reason.

\section{Qualitative discussion and validation}

In this part the velocity vectors and pressure coefficient contours are used to provide a good understanding of the flow field. 
As evident in Figure 3, the pressure difference is higher and this will lead to a stronger jet which will be explained later. The sudden changes in the rear portion of the damage hole are because of the local stagnation of the flow. The gap in the damage results was where the damage hole cut through the surface.

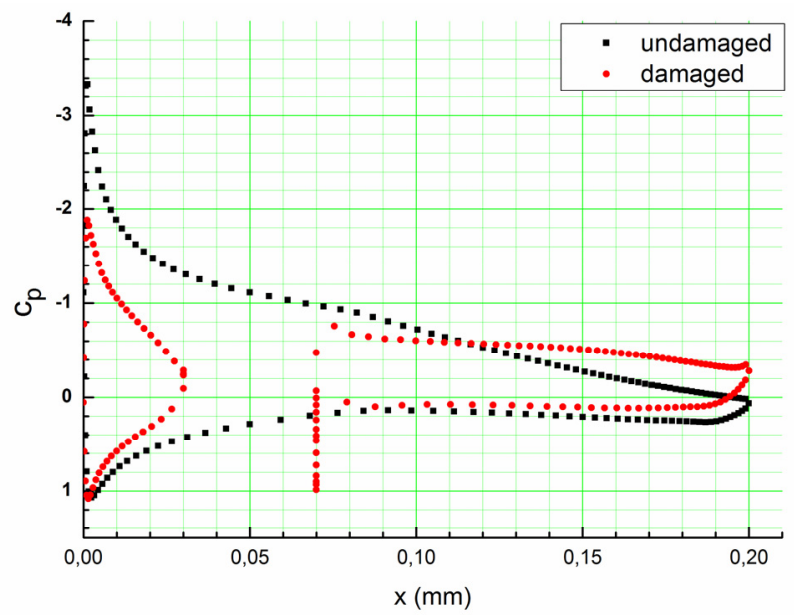

Figure 2: Wing centreline pressure coefficient $\left[20 \% \mathrm{c}\right.$ at quarter-chord for $\left.8^{\circ}\right]$.

Figure 3(a) shows the velocity vectors around the damaged aerofoil from lateral view for $0^{\circ} \mathrm{deg}$ incidence angle.

There is a weak jet exiting the damage hole which formed an attached wake behind the damage hole.

Because of the pressure difference between two surfaces of the aerofoil, the flow would like to penetrate from the lower surface of the aerofoil to the upper surface and this flow through the damage is pushed through the damage hole and added to the damage wake.

Figure 3(b) shows the velocity vectors of the numerical solution on the upper surface of the aerofoil. At the rear edge of the damage hole, there are two vortex centres, observed to be contra-rotating vortices approximately symmetrical about the hole centreline.

These vortices are the results of interaction between jet flow exiting the damage and free stream. The weak jet exited from the rear edge of the hole was immediately bent over, forming an attached wakes to the upper surface. When the incidence angle is increased higher than $0^{\circ}$ degree, the jet no longer immediately bent over upon exiting the hole and penetrated to the free stream. Finally a separated region is formed between the jet and upper wing surface.

Figures 4(a) and 4(b) that are for incidence angle of $8^{\circ}$, show the separated region and detached wake of strong jet.

It is noticeable that the stall angle of an undamaged aerofoil is normally $12^{\circ} \mathrm{deg}$ but for damaged aerofoil this angle shifted to $14^{\circ} \mathrm{deg}$. 


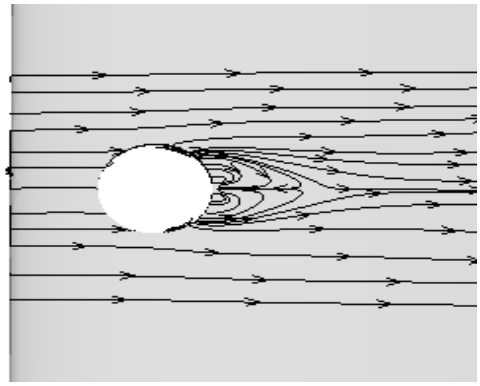

a) Upper view

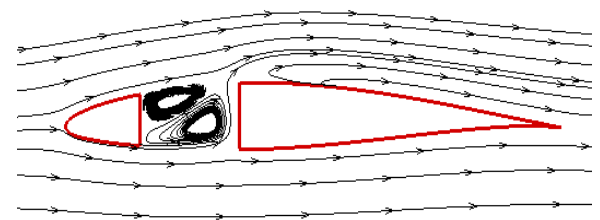

b) Lateral view

Figure 3: $\quad$ Velocity vectors for damaged aerofoil $\left(0^{\circ} \mathrm{deg}\right)$.

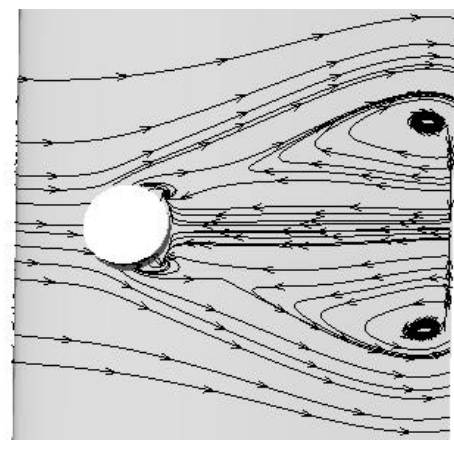

a) Upper view

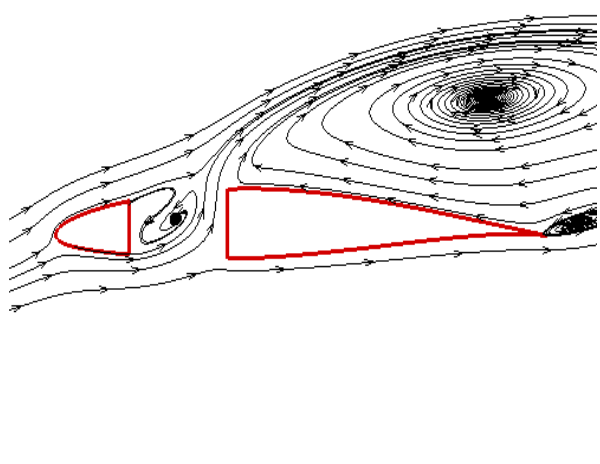

b) Lateral view

Figure 4: $\quad$ Velocity vectors for damaged aerofoil (+8 deg).

The above numerical results for the velocity vectors on the upper surface of the aerofoil are compared to the results of flow visualisation that was implemented by Render et al. [4] and Mani and Render [5]. In Figure 5 that is for damaged aerofoil, it represents experimental results. The results are presented for incidence angles of $0^{\circ} \mathrm{deg}$ and $8^{\circ} \mathrm{deg}$ that represent weak and strong jet respectively.

As evident, the general feature of the flow fields obtained by numerical analysis (figures 3 and 4) are in a suitable consistency with experimental results. It is clear in both flow visualisations that the width of the wake behind the damage hole increases with incidence angle because of the strength of exiting jet.

There are also two large surface wakes in $8^{\circ} \mathrm{deg}$ incidence angle behind the damage hole which are clear in experimental and numerical results. In both cases they are counter rotating vortices. 
Some phenomena such as laminar separation and transition and thus, the laminar bubble can not be captured by the software which are visible in the side of the damage hole in experimental results.

The differences between experimental and numerical results are due to numerical error associated with numerical solution.

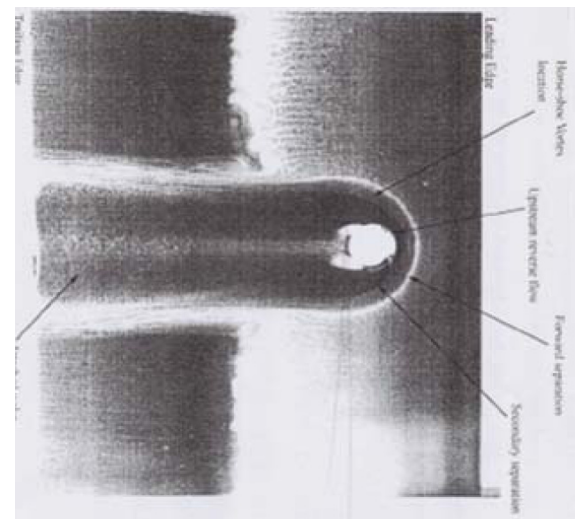

a) Weak jet

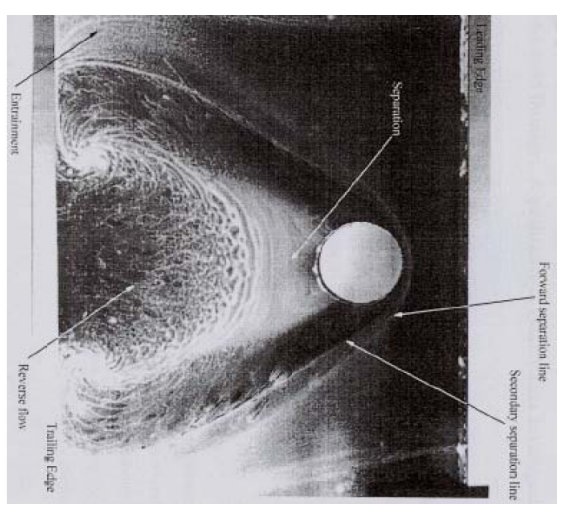

b) strong jet

Figure 5: $\quad$ Experimental flow field for damaged aerofoil at $0^{\circ} \mathrm{deg}$ and $8 \mathrm{deg}$ incidence angles.

\section{Quantitative discussion and validation}

In this section the quantitative results are investigated using $\mathrm{dC}_{\mathrm{L}}$ and $\mathrm{dC}_{\mathrm{D}}$, vs $\alpha$ diagrams. For a better perception and assessment of the effects of the damage; the corresponding above results are transcribed in terms of increments of losses of $C_{L}$ and $C_{D}$. The increments $\mathrm{dC}_{\mathrm{L}}$ and $\mathrm{dC}_{\mathrm{D}}$ are defined from the undamaged state as follows:

$$
\begin{gathered}
\mathrm{dC}_{\mathrm{L}}=\mathrm{C}_{\mathrm{L}_{\text {damaged }}}-\mathrm{C}_{\mathrm{L}_{\text {undamaged }}} \\
\mathrm{dC}_{\mathrm{D}}=\mathrm{C}_{\mathrm{D}_{\text {damaged }}}-\mathrm{C}_{\mathrm{D}_{\text {undamaged }}}
\end{gathered}
$$

Before studying the influence of damaged aerofoil, analysis on the undamaged model was done. The results obtained were found to agree reasonably well with those found in the literature [2].

This numerical procedure allows us to capture the stall phenomenon, for instance, the lift coefficient reaches a maximum value of 1.20 for an angle of stall of $12^{\circ}$. Also, the angle of zero lift is close to $-2.5^{\circ}$, which is consistent with this non symmetric profile. The coefficient of drag reaches a minimal value of 0.016 for the angle $0^{\circ}$. 
Now we consider the damaged aerofoil to explain the influence of the damage on the aerodynamic characteristics of the aerofoil and the effect of both diameter and chordwise position of damage expressed only in increments of lift and drag coefficients as explained above.

In figures 6 and 7, we present, simultaneously, the influence of both diameter and chordwise position of damage with the experimental and numerical curves. One can see an increase of the $C_{D}$ over most of the incidence range except close to the stall angle where part of the jet goes through the hole. The strength of the jet is in direct relation with incidence angle.

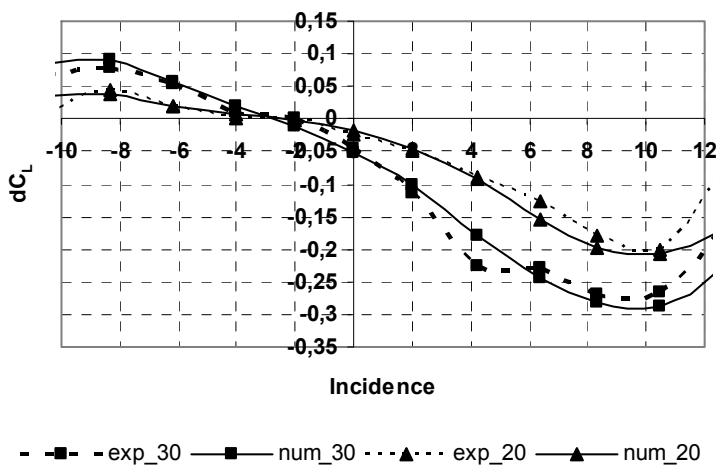

Figure 6: Influence of damage diameter at quarter chord on lift coefficient (experimental and numerical solutions).

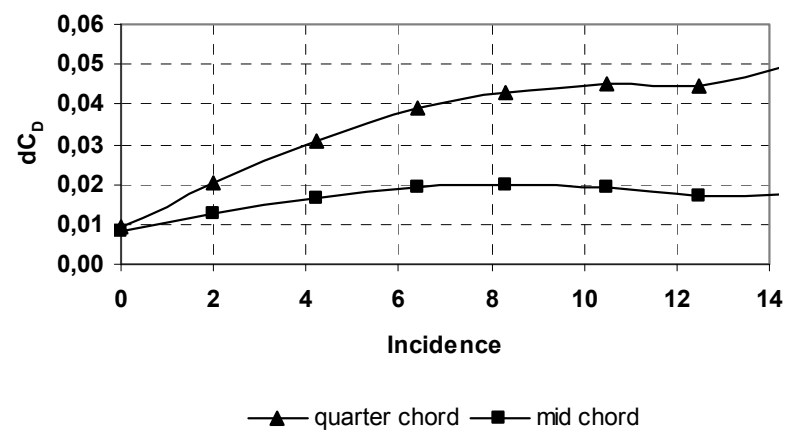

Figure 7: Chordwise influence of damage on drag coefficient (numerical results).

There are two mechanisms for the drag increase. For small angles of incidence, the attached jet increases friction drag while for higher angles of incidence, the strong jet forms a separated wake which increases form drag. An additional pressure drag is produced by the damaged hole which creates a positive pressure increment on the wing internal surface. 
Results also show a reduction in the amplitude of $\mathrm{C}_{\mathrm{L}}$ over the entire range of positive incidence angle. This reduction in $C_{L}$ is due to the hole through flow which affects the distribution of pressure at the upper surface.

Lift loss for the quarter chord damage is higher than the mid-chord damage. This is expected because when we approach the leading edge; the suction pressure on the upper surface is strongly reduced affecting consequently the lift coefficient. The drag increase is higher for the quarter chord damage, where the chord wise extent of the wake is greater than seen for the mid-chord.

For the effect of diameter, results show that lift increments $\mathrm{dC}_{\mathrm{L}}$ against incidence for different values of the diameter and over the positive incidence range $\left(\alpha>-2.5^{\circ}\right)$, an increase of the hole size results in a decrease of the lift coefficient.

This is expected because a larger damage size allows a greater through flow, and perturbs even more the pressure distribution at the upper surface. Increasing the hole diameter changes the jet shape from weak-jet to strong-jet.

The drag also increases with hole diameter over the entire incidence range. Indeed, an increase of the diameter increases the wake area size. In order to validate the numerical results and to assess that the numerical procedure is performed truly, the experimental coefficients are also required.

The aerodynamic coefficients for damaged aerofoil from numerical solution are compared to experimental coefficients of circle damaged aerofoil that were available in [2] and [3]. It is observed that the trends of experimental and numerical curves in the two figures are so similar to each other and there is a very good consistency between the curves. In the worst case, the difference between the values of $C_{L}$ and $C_{D}$ at the high incidence close to the stall is less than $10 \%$. In this incidence angle, the numerical error is at the highest value because of stall phenomenon and causes this difference.

The difference between numerical and experimental results that is observable in figure 6 is because of numerical error. Generally, the results show that the numerical solution has no major error in modelling and solving the equations.

The constant difference between two curves is originated from numerical sources such as turbulence modelling, boundary-layer capturing and truncation error. It can be concluded from this diagram that numerical solution has been performed truly and physical phenomena are correctly captured.

\section{Conclusion}

In this paper the various damages have been studied comprehensively by numerical investigation and the results have been validated using the relevant experimental results. The multi-block grid allows us to reduce time consumption and enhances consistency and accuracy. It is concluded that the damages (circle damage) give a reduction in lift and an increase in drag. These variations depend on the incidence angle and the diameter (jet exiting from the damage hole). The numerical simulation gives accurate results comparatively to experimental results. 


\section{References}

[1] Ball, R. E., The Fundamentals of Aircraft Survivability Analysis and Design, $2^{\text {nd }}$ ed., AIAA Education Series: Reston, VA, 2003.

[2] Irwin, A. J. \& Render, P. M., The influence of mid-chord battle damage on the aerodynamic characteristics of two-dimensional wings, The Aeronautical Journal, 104(1033), pp.153-161, 2000.

[3] Irwin, A. J., Render, P. M., McGuirk, J. J., Probert, B. \& Alonze, P. M., Initial investigation into the aerodynamic properties of a battle damaged wing. $13^{\text {th }}$ AIAA Applied Aerodynamics Conference, AIAA Paper No. 951845, 1995.

[4] Render, P. M., De Silva, S., Walton, A. J. \& Mani, M., Experimental investigation into the aerodynamics of battle damaged airfoils, Journal of Aircraft, 44(2), pp.539-549, 2007.

[5] Mani, M., \& Render, P. M., Experimental investigation into the aerodynamic effects of airfoils with triangular and star shaped through damage," AIAA Paper 2005-4978, June 2005.

[6] Djellal, S., \& Ouibrahim, A., Aerodynamic performances of battle-damaged and repaired wings of an aircraft model", Journal of Aircraft, 45(6), pp.2009-2023, 2008.

[7] Saaedi, M., Ajalli, F. \& Mani, M., A., Comprehensive numerical study of battle damage and repairs upon the aerodynamic characteristics of an aerofoil, The Aeronautical Journal, 114(1158), August 2010.

[8] Djellal, S., Effects of Battle Damage Repairs on Aerodynamic of a wing, Sabbatical leave report, Aeronautical and Automotive Engineering Department, Loughborough University, 2004. 